\title{
Filigrane
}

Écoutes psychothérapiques

\section{Dubo... Dubon... Dubonnet. Lecture des problématiques alcooliques aux regards des faits de société}

\section{Nicolas Peraldi}

Volume 17, numéro 2, automne 2008

L’avenir du clinicien II

URI : https://id.erudit.org/iderudit/019424ar

DOI : https://doi.org/10.7202/019424ar

Aller au sommaire du numéro

Éditeur(s)

Revue Santé mentale au Québec

ISSN

1192-1412 (imprimé)

1911-4656 (numérique)

Découvrir la revue

Citer cet article

Peraldi, N. (2008). Dubo... Dubon... Dubonnet. Lecture des problématiques alcooliques aux regards des faits de société. Filigrane, 17(2), 144-163.

https://doi.org/10.7202/019424ar
Résumé de l'article

Le travail au quotidien avec des personnes en situation d'exclusion ouvre des voies de questionnement multiples sur le rapport que la société entretient avec ce « produit » si particulier qu'est alcool. En partant des problématiques psychiques et des impasses du sujet en désocialisation, au travers des manifestations symptomatiques et de différentes acceptations du réel, l'auteur cherche à mettre en évidence les maillages sociaux dans lesquels sont imbriquées ces personnes trop communément étiquetées d'alcooliques. C'est dans les plis du langage, dans ce processus qualifié d'oubliance, qu'il cherche les traces d'un désir qui se manifeste sans modération. 


\title{
Dubo... Dubon... Dubonnet. Lecture des problématiques alcooliques aux regards des faits de société
}

\author{
nicolas peraldi
}

Le travail au quotidien avec des personnes en situation d'exclusion ouvre des voies de questionnement multiples sur le rapport que la société entretient avec ce «produit» si particulier qu'est alcool. En partant des problématiques psychiques et des impasses du sujet en désocialisation, au travers des manifestations symptomatiques et de différentes acceptations du réel, l'auteur cherche à mettre en évidence les maillages sociaux dans lesquels sont imbriquées ces personnes trop communément étiquetées d'alcooliques. C'est dans les plis du langage, dans ce processus qualifié d'oubliance, qu'il cherche les traces d'un désir qui se manifeste sans modération.

«L'alcool tue lentement. On s'en fout, on est pas pressés »

(Perrier, 1982, 14)

$\mathrm{L}$

orsque j'étais enfant, mes parents m'emmenaient parfois faire des courses dans Paris en métro. C'était un endroit extraordinaire pour le petit garçon que massait dans des wagons, et à travers les vitres je voyais se dérouler des affiches massait dans des wagons, et à travers les vitres je voyais se dérouler des affiches publicitaires, notamment celle de la marque Dubonnet.

À l'époque, je ne savais pas encore à quel produit cela faisait référence. Mais j'entendais bien la gêne de mes parents lorsque je lisais à voix haute ces mots, dans leur construction, leur espace et leur aboutissement. Ce rythme résonne encore dans mes souvenirs comme une mélopée scandée par les arrêts de la rame et le bruit des rails : Dubo, Dubon, Dubonnet...

Ce n'est que plus tard, que j'ai compris à quoi cela faisait référence. Je ne l'ai d'ailleurs compris qu'à partir du moment ou ces affiches ont progressivement disparu des tunnels du métro. Dubonnet était la marque d'un apéritif alcoolisé. Pas n'importe laquelle semble-t-il. On la voyait dans des lieux très fréquentés. Elle s'affichait au vu et au su de presque tous. Elle avait une place privilégiée dans la cité. Les quelques annonces qui suivent nous montrent encore une fois comment la publicité — on disait alors «la réclame» — savait jouer des habitudes, et s'offrir à chacun comme faisant partie de son «ordinaire»; c'est d'ailleurs ainsi que l'on appelait le vin de table, étoilé s'il vous plaît ${ }^{1}$ ! 
C'est avec ces représentations-là que nous travaillons encore aujourd'hui lorsque nous avons à faire avec un sujet souffrant de ce qu'il est maintenant convenu d'appeler «la maladie alcoolique ».
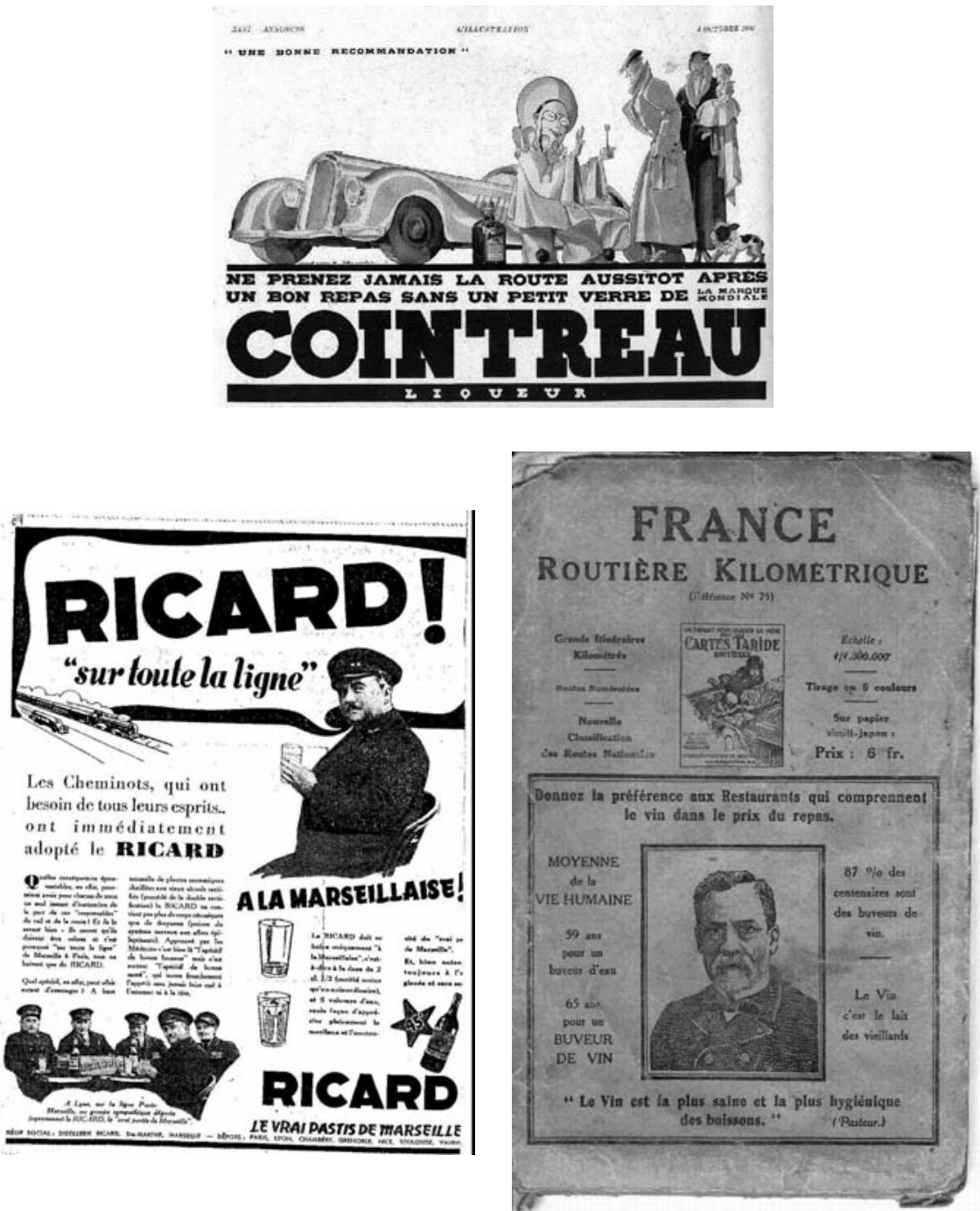

À traiter ici un tel sujet, il me sera nécessaire d'éviter les écueils du Charybde de l'exclusion sociale et du Scilla de l'alcoolisme. Les hommes et les femmes que je rencontre se jouent de ces deux problématiques. Ils se développent dans ce double espace, dans l'écart qui s'y creuse. Je situe en partie mon travail en amont 
de la pathologie alcoolique et des différentes lectures diagnostiques qui peuvent parfois clore toute écoute, ou du moins en délimiter l'espace. L'alcool apparaît au carrefour des fonctionnements psychiques. Il fait ouverture ou ornière à toute tentative de psychologisation. Tantôt signe d'une psychose franche, il devient l'objet derrière lequel court le névrosé pour échapper à son désir. La difficulté s'accentue plus encore lorsque les processus de désocialisation se développent. On ne peut pas affirmer qu'il existe une catégorie homogène d'exclus, et l'on ne peut pas non plus faire un nouvelle nosographie (avec sa posologie) liée aux phénomènes de l'exclusion. Il serait dommageable de prendre les symptômes pour ce qu'ils ne sont pas. Ils expriment quelque chose du malaise dans lequel ces personnes vivent. On retrouve chez ces personnes en exclusion une intolérance à la frustration, une insécurité intérieure, une hypersensibilité à toute remarque ainsi qu'un sentiment de vacuité et d'ennui. Ces personnes passent très facilement à l'acte pour résoudre des tensions internes, ce qui, on l'imagine aisément, entraîne des conduites professionnelles ou affectives instables, parfois même autodestructrices. C'est souvent le signe de formations traumatiques, dans lesquelles la question qui se pose n'est pas celle du refoulement de souvenirs ou de certaines représentations, mais plutôt de l'impossibilité d'inscrire un événement dans les processus de la représentation et de la pensée. Il n'y a pas de souvenir à proprement parler, mais une trace psychique d'un «quelque chose » à l'état brut, quelque chose qui a eu lieu, et qui se traduit par des actes compulsifs, hors de toute réminiscence. On voit bien que, dans le cas de personnes dont le sentiment de sécurité a été perturbé, brisé ou perdu par suite d'événements graves ayant bouleversé leur environnement, un besoin de sécurité permanent reste en attente. Les personnes en exclusion attendent «la peur au ventre» que l'on vienne les chercher, qu' on leur propose quelque chose, qu'on réponde à leurs angoisses. Elles attendent en buvant pour remplir cet abîme qu'est l'angoisse. Elles se confrontent à un « réel $^{2} »$ tel que l'alcool ne fait pas nécessairement partie de leur préoccupation ou de leur questionnement. Il est là. C'est un objet avec lequel, par lequel, et parfois pour lequel elles vivent. C'est un point de fixation dans le réel dans leur histoire, qu'il est difficile de symboliser. La place que je tiens auprès d'elles, celle d'un psychologue d'un $\mathrm{CHRS}^{3}$, me permet d'interroger l'alcool sans pour autant en faire une spécificité. Mon champ d'intervention, tout comme le positionnement qu'il induit par rapport à cette problématique, n'est pas limité comme pourrait l'être la pratique d'un psychologue dans un service d'alcoologie ou de psychiatrie.

Roger Gentis, il y a quelques années déjà, sur un ton militant mais toujours d'actualité, dénonçait avec force la stigmatisation des alcooliques sur le registre médical, psychiatrique et bien évidemment celui de l'économie sociétale.

«Prenez l'alcoolisme par exemple. Ca fait déjà quelque temps qu'on se doutait que c'était un problème psychiatrique, on avait découvert que c'était pas un vice comme on croyait au moyen âge mais une maladie, les buveurs eux-mêmes ils étaient pas 
fâchés que ça soit une maladie, comme ça ils pouvaient dire qu'ils étaient guéris quand ils ne buvaient plus, enfin tout le monde était bien d'accord et on se disait que puisque c'est une maladie, à mesure qu'on la connaîtrait mieux on pourrait mieux la soigner et que comme ça on finirait bien un jour par assécher tous les buveurs, ça ferait comme le Zuiderzee et ça serait autant de gagné pour la pays et le produit national.» (Gentis, 1971, 15)

Cependant l'on s'est aperçu de l'écart qui existe entre le «malade » alcoolique et la pratique psychiatrique, surtout de nos jours où cette dernière se réduit comme peau de chagrin.

«Ce qui fait que maintenant on se dépêche de sortir les alcooliques des hôpitaux psychiatriques et de leur construire des petites maisons de cure pour eux tout seuls, comme ça, ça se passera en famille et ça sera-t-il plus gentil - à moins que les banquiers, qui s'intéressent eux aussi à la psychiatrie, ils calculent que la cure des alcooliques c'est d'un bon rapport, alors évidemment l'alcoolisme aura vite fait de redevenir un problème psychiatrique» [mais] «de toutes façon y a pas des risque qu'on aille trop loin et que l'on soulève des problèmes qui toucheraient de trop près à l'ordre social.» (Gentis, 1971, 17)

L'alcool est de l'ordre du symptôme, d'une parole d'un psychisme en souffrance, pour reprendre cette notion spécifique à la clinique de la désocialisation développée par Sylvie Quesemand Zucca. Les personnes que je rencontre présentent pour la plupart de réelles difficultés avec ce produit en vente libre. Si j'insiste d'emblée sur la réalité des faits et sur la polémique économique qui en résulte, c'est parce que c'est l'essentiel du propos que je souhaite développer.

Lorsque je signifie le réel rencontré, c'est justement parce que cette inscription prend une place prépondérante dans la société et dans la vie des personnes ayant un parcours de rue et d'errance plus ou moins long.

Le réel à partir duquel je travaille n'est bien sûr pas la réalité telle qu'elle est communément admise, bien qu'il s'y déploie. Le concept de réel en psychanalyse est assez complexe à utiliser. Freud n'a pas évoqué cette notion, parlant plus aisément du couple réalité extérieure - réalité psychique. Dans sa refonte de la théorie freudienne, Lacan a inauguré une grande partie de son enseignement avec sa présentation sur Le symbolique, l'imaginaire et le réel (Lacan, 1953). Au fur et à mesure des séminaires, le réel à changé de signification. De plus, les différents psychanalystes développant cette notion ne sont pas forcément d'accord sur une définition. Il suffit de lire les différents dictionnaires ou vocabulaires existants pour mesurer l'écart entre chaque occurrence. Elles ne sont d'ailleurs pas en soi antinomiques. Elles révèlent différentes possibilités de lecture d'un sujet pris dans 
les affres d'un symptôme auquel il ne comprend rien. Le réel est ce qui existe avant le sujet, et dans lequel il s'inscrit selon des modalités qui lui appartiennent. C'est un lieu de rencontre entre la réalité psychique d'un sujet désirant et sa perception de la réalité extérieure où son désir vient se développer, à son grand dam parfois. Il semble qu'un livre entier ne suffirait pas à mettre en articulation, à explorer, les différentes lectures du réel, tant chez Lacan que chez ses élèves. Tous néanmoins reconnaissent le caractère privatif du réel. Je me référerai essentiellement pour cette élaboration à sa première mouture, parce qu'elle est plus proche de ma pratique, de mon acceptation et du sujet que je souhaite développer.

Le réel, c'est aussi celui de la bouteille qui circule de main en main, de maladies en maladies, de la mort à la mise en bière.

Et, c'est enfin, et ce n'est pas le moindre, celui de la société de consommation qui s'offre là pour le sujet qui s'y acoquine comme une tentative désespérée, une fonction de «briseur de soucis», selon l'expression de Sigmund Freud (Freud, 1929, 21). Trois réels, inscrits dans différentes grilles de lecture, qui prennent corps, du sujet au collectif, de la culpabilité à la responsabilité.

Je vais m'autoriser tant que possible à développer ces trois lieux pour tenter de les mettre en mouvement, de les articuler les uns par rapport aux autres, et de saisir ce qui peut parfois être mis en oeuvre avec ces personnes qui se lient à l'alcool comme pour mieux se délier du langage.

\section{I}

La clinique des phénomènes de clochardisation nous entrâne à baisser les bras, à lâcher prise avec le fantasme de croire qu'on pourrait, pétri de bonne volonté, aider ces «pauvres hères ${ }^{4}$ » à s'inscrire dans le socius autrement que dans cet état de déliquescence.

Le réel, quelles que soient ses acceptations, s'ordonne donc par son caractère privatif, échappant à l'Imaginaire et au Symbolique. C'est ce qui amène Lacan à le définir comme l'impossible. C'est ce contre quoi bute le sujet. Il existe au préalable, et conditionne les mesures de l'existence d'un sujet. Le réel fait obstacle au principe de plaisir, c'est un heurt. L'important est, de fait, de mesurer comment un sujet s'y confronte, comment il s'en arrange et comment il le met en mouvement autour de lui. Cet impossible auquel il se heurte souvent violemment, peut devenir un mode de vie et ainsi avoir des effets non négligeables pour celui ou celle qui intervient dans ce réel.

C'est par exemple l'impossibilité de travailler comme on nous l'a appris pendant des années. Les travailleurs sociaux le mesurent chaque jour, le personnel soignant s'y confronte avec dépit, et les psychologues y perdent leurs références. C'est là un des premiers effets de ce type de pratique, il faut une dose de masochisme pour aller s'occuper, à sa façon, de ces personnes qui mettent en échec nos tentatives professionnelles, qui se jouent de nos stratégies, et qui nous attendent ivres le matin, pour mieux nous montrer que tout ce que nous avons fait avec elles la veille est dilué une nouvelle fois dans du Camberlieu à 1,2 euros le litre en 
bouteille plastique. Elles nous font revivre ce qu'il en est pour elles d'un état de détresse infantile non symbolisé, rejoué au jour le jour dans la relation à l'autre, pour vérifier à la fois leur propre «désaide» et les effets que cela suscite chez l'autre, tout aussi impuissant. Elles recherchent et retrouvent en l'autre le trauma éprouvé mais pas vécu, pour reprendre la formulation de D.W. Winnicott (Winnicott, 2000, 209).

Dans le discours de ces personnes à la rue, les références à l'alcool viennent enrayer toute symbolisation. C'est en cela que l'alcool vient comme un point de butée et de jouissance. L'alcool maquille, «comme une voiture volée» dit l'expression. Rien n'est pourtant plus visible. C'est un point de butée donc, un semblant de réel duquel l'on ne peut se détacher. Le buveur a besoin de boire pour se sentir exister et mourir en même temps. Le soignant et le social (des appellations pour délimiter et circonscrire le champ de l'autre) ont besoin de l'alcool pour se mettre au travail, pour trouver un sens à leur acte. L'alcoolique à la rue se donne à voir, et à boire si je puis dire, et ce jusqu'à la lie. Il ne s'arrête pas. C'est le lieu de sa folie, cette inquiétante étrangeté, cette incompréhension qu'il (re)trouve dans le regard de l'Autrel'écart qui existe entre soi et la perception de soi. Désirs et fantasmes se lient dans cette réalité inaccessible à toute pensée. L'alcool est là. Personne ne peut y déroger, tout le monde y est rappelé au quotidien. L'essentiel reste de ne pas

«s'adapter au réel plus ou moins bien défini ou bien organisé, mais de faire reconnaître sa propre réalité, autrement dit son propre désir, [...] le faire reconnaître par ses semblables : c'està-dire de le symboliser » (Lacan, 1953, 48).

Il y a donc «un possible» à ce réel, en cela qu'il permet justement de penser autrement, de se donner du temps et de jouer avec ces «malades » alcooliques des différentes modalités de prise en charge. Le jeu, c'est de cela dont manquent ces personnes. Elles en souffrent à tel point qu'elles le cherchent dans toutes les relations qu'elle entretiennent. Jouer, c'est nouer. Nouer, c'est mettre du lien là où il n'y en a souvent plus, c'est accorder des capacités à soi et à autrui.

\section{II}

L'acte est très présent dans la clinique de la désocialisation. L'acte du professionnel déjà, pas toujours adapté, mais toujours signifiant de la place qu'il essaye de tenir auprès ou contre ces personnes. Et surtout l'acte de l'exclu qui se donne à voir comme un déchet posé sur le trottoir, de cette chose qui a chu et qui n'a pas été suffisamment bien reçue.

Madame A. a été admise dans le centre d'hébergement après une longue période d'errance. Les hivers successifs et l'alcool ont eu raison de ses résistances. Seule, Madame A. a renoncé, elle a demandé à être prise en charge. Ce renon- 
cement a un prix: celui de consentir à la vie en collectivité, celui de respecter les règles du CHRS, celui de s'engager dans des démarches dites de réinsertion.

Mme A. a toujours été soumise aux désirs des hommes qui ont ponctué sa vie. Son père d'abord qui travaillait à la mine. Lorsqu'il rentrait, après être passé au bistrot, il était fatigué et ne supportait pas les bruits que ses enfants pouvaient faire. Il fallait «se tenir à carreau », sinon la main savait être leste. Mais qui aime bien châtie bien, comme elle dit, comme on lui disait. Son mari ensuite. Elle l'a rencontré à 17 ans. Une soirée de bal, un peu trop arrosée, et l'affaire était conclue. Ils se sont mariés rapidement, alors qu'elle était enceinte. Quelques enfants plus tard, elle n'a plus supporté ce qu'il lui faisait endurer. Mais elle ne s'étend pas sur le sujet. Pour la première fois de sa vie, elle a pris ses affaires, et elle est partie. Commence alors "une drôle de balade». Elle rencontre Rémi. Il était sans famille (le prénom comme une assignation à l'Etre?), elle n'en avait plus. Ils ont vécu d'expédients divers, de mendicité et de quelques allocations grappillées au gré des administrations. Gare du Nord, ils ont rencontré une petite bande qualifiée de Sans Domicile Fixe. Là, les choses ne se sont pas arrangées. La santé de Madame A. a commencé à se dégrader. Phlébite sur phlébite, elle a de plus en plus de mal à marcher. Pour contrer la douleur, elle boit. Rémi veille sur elle, il lui fournit ce dont elle a besoin. 5 ans passent. Ils vont parfois se réchauffer dans un centre d'urgence. D'autres fois, ils squattent des immeubles abandonnés ou en construction. On est vraiment là dans une représentation du corps. Mais, l'état de santé de Madame A. empire. En janvier, alors que Paris est recouvert de neige, elle est hospitalisée. Le Samu Social a réussi à la convaincre de céder (s'aider) un peu. Rémi, lui, refuse toujours d'être pris en charge. Il reste à la rue et lui dit qu'il l'attendra. Quand elle sort de l'hôpital, 2 semaines plus tard, quid de Rémi. Il n'est pas au rendez-vous. Leurs comparses ne l'ont pas vu depuis quelques jours. Au dernière nouvelle, il toussait beaucoup. Le week-end passe, et Rémi n'est toujours pas là. C'est par hasard, s'il existe, que Madame A. apprend qu'il est décédé. Rémi est mort pendant une nuit particulièrement froide. Pierre, un vieil ami, était là quand les pompiers sont venus le chercher. Il a été enterré au Mont Valérien, comme beaucoup d'autres morts dans des circonstances similaires. Madame A. n'en peut plus. Elle cède encore une fois, et demande un hébergement pérenne, du moins stable. Elle veut «rompre» avec son parcours d'errance et se reposer. Au centre d'hébergement, elle retrouve quelques amis. Certains ont eux aussi arrêté la rue. Ils s'en sont sortis comme ils disent. D'autres vagabondent dans le voisinage. Parmi eux, Eddy. Lui, il est toujours à la rue. Il ne peut pas avoir le RMI parce qu'il a perdu sa carte d'identité. Et puis il ne veut pas en refaire parce qu'il faut aller au commissariat, et qu'après sa vingt et unième incarcération il a décidé qu'il ne parlerait plus jamais avec un policer. Une façon comme une autre de ne pas avoir à "retourner au ballon ${ }^{5}$ ». Par conséquent, il n'a pas de revenu. Madame A. se charge donc de le fournir en alcool, en tabac et de l'héberger. Lorsqu'elle peut, en début de mois, ils vont passer une nuit ou deux dans un hôtel. Elle l'aime, et c'est son droit, même si cela lui coûte. Et, elle le 
paye. Lorsqu'elle a des sous, il les lui prend pour aller leur chercher à manger et à boire. Elle attend, et la nuit est tombée elle rentre se coucher. Le lendemain quand il revient, il ne va pas bien. Il a l'alcool mauvais dit-elle comme pour l'excuser du cocard qu'elle porte. Quand il est là, tout va bien. Elle est contente, elle se sent protégée. Elle peut boire en paix. Quand il est absent, la plupart du temps, elle pleure, la bouteille à portée de main, dans son sac accroché derrière son fauteuil roulant. Mais elle l'aime, et cela n'a pas de prix, même si souvent elle n'a «que ça» dit-elle en s'accrochant à son litre de vin rouge.

«Je n'ai que ça.»

Ceux qui travaillent avec des personnes présentant des pathologies alcooliques entendent souvent cette phrase qui tonne comme une sentence à toute démarche de soins ou d'accompagnement. Ceux qui travaillent avec des personnes à la rue y sont aussi confrontés, puisqu'un des faits de l'exclusion est une forte consommation d'alcool. Il est impossible de ne pas prendre en compte cette réalité à laquelle ces hommes et ces femmes nous confrontent. L'exclusion a une histoire. Elle s'inscrit dans différentes trajectoires individuelles où l'on repère, comme des balises sociales, des impasses subjectives similaires. Il est notoire que les personnes qui vivent à la rue boivent. C'est presque une lapalissade. Mais elles ne boivent pas toutes pour les mêmes raisons. J'y reviendrai ultérieurement. L'alcool n'est pas le fait ou le symptôme d'un fonctionnement psychique particulier. Il prend la place d'un objet à un moment $\mathrm{T}$ de l'histoire d'un sujet, jusqu'à ce que, justement, objet et sujet se confondent l'un et l'autre, se diluent l'un dans l'autre. L'autodestruction liée à l'utilisation ou à l'abus d'alcool peut être conçue comme une tentative visant à se débarrasser de certaines expériences pénibles et perturbatrices par le biais de l'engourdissement, de la tranquillisation et de l'euphorisation. L'alcool vient là noyer le sujet dans l'illusion d'un «hors langage». C'est une forme d'automédication devant le déferlement anxiogène de l'excitation non liée. Le moi tente d'évacuer les perturbations de l'appareil psychique, de rétablir un état de quiétude, proche de l'inorganique. Il cherche des moyens à disposition à l'extérieur pour faire taire le trauma.

La bouteille circule comme un objet commun qui ferait lien entre eux. Ils se la partagent. Les uns financent, les autres l'achètent, et tous boivent. C'est comme au bistrot, le jeu est de savoir qui va commander, qui va payer pour que tous trinquent. Mais ce lieu où ils se retrouvent n'est pas un lieu de convivialité. Ils ne sont pas là pour apprécier un moment ensemble. Ils y sont parce qu'ils nous adressent un message, silencieux et énigmatique: «Regarde, toi qui passes. Regarde si tu l'oses!» S'arrêter là, c'est indéniablement prendre le risque de devoir parler de l'alcool. Il serait d'ailleurs dommageable d'en faire l'économie puisqu'ils nous le donnent à voir, dans sa présence comme dans ses effets. Mon expérience professionnelle, et les limites abordables du bon sens, me font dire qu'à ne pas vouloir parler de la présence de l'alcool - ce qui n'est pas pour autant focaliser toute son attention dessus - c'est créer les conditions pour que cet objet vienne intoxiquer 
toute la relation, qu'elle soit éducative ou thérapeutique. Un «faire comme si » parfois demandé comme une provocation, comme une marche de plus vers la déchéance, et qui s'inscrit dans la continuité d'un abandon de soi avec l'autre supposé désespéré.

Dans les entretiens que je peux avoir, l'alcool n'est que très rarement un problème. Il n'apparaît d'ailleurs que très ponctuellement dans leurs discours. Et toujours avec modération. Un faux-semblant, un mi-dit, qui crée une boiterie dans le suivi, laquelle, comme toute boiterie, a pour fonction de ne pas se dégager de son rapport à l'exclusion, de se maintenir au ban. Lorsque j'écris qu' «il» n'apparaît que très ponctuellement, je me dois de développer mon propos. Bien évidemment, il est très présent, soit explicitement, soit implicitement. Tous les résidants que je vois «parlent» d'alcool, et ce de différentes façons, mais il ne m'en parle pas. Tel ce résidant ${ }^{6}$ qui vient tous les lundis matin depuis plusieurs années, sans avoir bu, parler de tout ce qu'il va boire après notre entretien. À chaque semaine, la même rengaine. «J'en ai marre de parler d'alcool» dit-il avant d'aller se «tisanner». Il parle d'alcool, certes, mais il ne dit rien de ces contrées dans lesquelles il voyage tous les jours depuis plus de trente ans. Il parle d'alcool pour ne pas parler de lui. Tel autre résidant, l'haleine chargée d'une odeur anisée, vociférant que jamais il n'a bu. Il ne déni pas pour autant, il montre c'est tout. Et puis, «il» c'est aussi l'Autre, celui à qui s'adresse ce boire, celui dont on ne parle pas. Là encore, il parle d'alcool pour ne pas parler de lui.

Dans la galerie qui les mène au centre d'hébergement, dans cet entre-deux où ils s'installent, ils se consacrent à la boisson comme à un sacerdoce. Ils boivent parce qu'ils ne vivent plus que ça, parce qu'ils ne savent plus comment nous montrer ce qu'ils pensent être. Une fois que quelqu'un a entendu maintes et maintes fois qu'il était alcoolique, comment faire pour le dégager de cette représentation, de cette place qui lui a été assignée, qu'il s'est assignée, dans et par le regard de l'autre?

«Je n'ai que ça» donc. Cette phrase qu'ils nous servent est préconçue, répétée, et signe parfaitement ce que ces drôles de personnages veulent nous montrer, ce qu'ils attendent de nous.

«Préconçue» parce qu'elle ne veut rien dire en soi. Elle abrase toute élaboration de sa problématique, elle clôt toute discussion possible. La vie à la rue a ses effets de déstructuration du langage au point qu'il se ferme. Il y a un processus d'oubli du langage qui n'est pas dû au hasard, ni aux alcoolisations massives.

«Répétée», en cela qu'elle est véhiculée par tous et à plusieurs reprises comme un thème commun à leur histoire. La singularité perd de sa force face aux divers processus d'exclusion. Cette phrase, c'est souvent la dernière qui leur reste, la dernière dont ils usent et abusent sans ménagement. Une parole creuse, qui amène à penser à la fois la béance qu'il y a entre les résidants et les professionnels, mais aussi le vide auquel les résidants se confrontent tous les jours.

Et «parfaitement» parce que justement, le paradoxe c'est ce qu'elle révèle dans son non-sens. Cette phrase est toute en négation. On passe du personnel à 
l'indéfini. «Je»s'efface devant «ça». Ce n'est plus le «je» de la psychanalyse. C'est une abrasion psychique dans laquelle l'individu se dilue dans l'alcool. «J'ai ça» affirmerait quelque chose d'un résidant - je n'ose pas encore parler de sujet —, de ce qu'il pourrait dire de sa place et de son rapport à l'alcool, à un objet. Il y aurait un avoir, un «au moins». Là, il n'y plus que «ça». Mais «ça», c'est quoi?

Pour le moraliste - ce dont les institutions ne manquent pas - c'est bien sûr l'alcool, si ce n'est la bouteille. Là, pas de problème, on confisque, on se lave les mains dans un grand discours sur les méfaits divers et variés de ce produit. On explique, force détails culpabilisants à l'appui, ce qui va arriver au résidant s'il continue à boire. On ne voit rien, on ne comprend rien, mais on «sadise» tranquillement la relation. Tout est question de volonté, disent ces bien-pensants.

Pour d'autres, «ça» c'est tout le problème. Il nous renvoie de plain-pied dans ce que vivent ces personnes, dans cet indéfini auquel elles se confrontent, cette chosification qu'elles endurent. Elles sont loin de la compensation d'un manque affectif, ou en tous cas, elles n'y sont plus. «J'avais la place de ça en moi » écrivait Marguerite Duras.

Si un signifiant renvoie à un autre signifiant, il est là nécessaire de prendre en compte tous les aspects que ce «ça» peut revêtir. Parce que c'est justement une question d'habillage, un habillage langagier à effeuiller pour comprendre ce qu'il voile. Ce «ça» donc, c'est l'objet, celui qui fait symptôme. Ce «ça» du réel de la consommation, qui prend forme dans la bouteille pour s'incarner dans le corps, c'est cet espace étranger qui enraye le lien symbolique, et qui met à mort. Parce que la finalité de ces cohortes qui envahissent nos métros - à tel point que la RATP a, semble-t-il, fait appel à un cabinet de designers compétents pour refaire de nouveaux bancs sur lesquels on ne peut pas s'asseoir et encore moins s'allonger - c'est qu'elles meurent dans d'effroyables conditions. Elles meurent seules, loin du regard, et sans sépultures. Au prix du mètre carré à Paris et dans sa banlieue, même les cimetières doivent être rentabilisés. C'est à tel point honteux qu'une association a dû se créer pour défendre les morts de la rue, pour faire en sorte qu'ils puissent avoir un enterrement. La société ne fait même plus son office de sépulture. L'alcool vient s'inscrire dans leur corps jusqu'à nous dire dans le silence du trépas, que nous n'avons pas entendu leurs hurlements de bêtes assoiffées par le besoin d'amour et le désir de reconnaissance.

\section{III}

Une petite histoire pour venir ici étayer la polémique que je souhaite ouvrir avec ces agencements du réel. Lorsque j'ai commencé à travailler avec ces personnes dites «SDF», je suis allé consciencieusement faire un test comparatif. À l'heure où tout se teste, pourquoi y aurais-je échappé?

En face de la structure d'hébergement dans laquelle je travaille, se trouve un magasin de grande distribution, dont je tairai le nom pour ne pas faire de publicité. Non loin de chez moi, se trouvait aussi une enseigne du même nom. Je suis donc 
allé voir si les produits vendus étaient les mêmes. C'était à s'y méprendre. La construction était similaire, les agencements identiques et les produits ne se démarquaient pas d'un lieu à l'autre. La simple différence, et non la moindre, était la quantité. Là où le supermarché de mon quartier avait laborieusement stocké une dizaine de bouteilles de vin blanc de (très) bon marché à défaut d'être de qualité, dans le magasin qui faisait face au CHRS, réputé pour accueillir des personnes présentant des pathologies alcooliques, étaient entreposées à perte de vue des palettes dudit vin laissant prévoir qu'ils ne seraient pas en rupture de stock. Ils ? Les boutiquiers étaient à 1 évidence soucieux de bien faire leur métier et de penser à leurs consommateurs, y compris «les clodos», comme on les appelle, puisque leur vide sera toujours comblé, ne serait-ce que momentanément, par des litres et des litres de vinasse «chairement» achetée ${ }^{7}$. «À celui qui est privé de tout, il est interdit de ne pas aimer la merde» écrit Beckett.

Que la société est bien faite. Elle pense à ses pauvres, et dans notre belle économie de marché, si vous ne pensez pas à vous, d'autres le feront. Si chaque gérant peut faire en fonction de la population qui réside aux abords de son magasin, et gérer ses stocks, puisque c'est son métier, je ne m'attendais pas pour autant à une telle profusion de vin au litre, à une telle satiété d'alcool, à une telle mise en abîme de ce que le marché pense du consommateur.

Don Quichotte contre les moulins à vent ${ }^{8}$. Il est très malaisé de travailler avec les personnes à la rue la question de leur dépendance, lorsque sans avoir forcément besoin de traverser la rue, l'alcool leur tend les bras, le pinardier répond au dilemme. $\mathrm{Au}$ «être ou ne pas être alcoolique que certains peuvent se poser, la réponse est inscrite en petit sur une étiquette. N'y entendez pas l'idée absurde de vouloir prohiber l'alcool, idée véhiculée par certains à la mémoire courte et la morale grandissante. La naïveté serait de croire que s'il n'y avait pas d'alcool, il n'y aurait pas d'alcooliques. Et, il n'y a rien de pire que de vouloir interdire. C'est la condition par excellence de toute transgression. Comme le disait si justement François Perrier,

«il n'est d'interdit qui ne crée le désir comme transgression.» (Perrier, 1982, 26)

Mais de là à créer les conditions d'une telle déchéance en masse, on peut se poser quelques questions. C'est toujours dans un rapport étroit entre la loi et la jouissance que se situent les personnes qui présentent une symptomatologie alcoolique. Et, c'est là que la société intervient dans le réel, voir comme réel. D'autres auteurs en parleront mieux que $\operatorname{moi}^{9}$, mais le praticien que je suis, abonné à la clinique de la désocialisation, ne peut que mesurer les ravages que cette grande dispensatrice de jouissance qu'est devenue l'économie de marché peut créer sur les individus; lorsqu'ils arrivent à le rester. Parce que c'est bien d'individu qu'il s'agit. De cette «personne considérée isolément par rapport à une collectivité» dixit le dictionnaire. Le travail imaginable est dès lors de faire en 
sorte que ces hommes et ces femmes à la rue depuis de nombreuses années ne se fondent plus dans la masse des SDF, pour redevenir une personne qui ne boit pas comme tout le monde. C'est à cette phrase que tout professionnel à affaire.

- Question : comment buvez vous?

- Réponse : comme tout le monde!

Ça laisse pantois...

Avant de vouloir qu'ils arrêtent de boire, l'abstinence étant considérée comme le remède à leurs maux, il faudrait déjà qu'ils se démarquent individuellement dans leur consommation et que chacun d'eux reconnaisse où il en est. On ne boit jamais comme tout le monde, on boit toujours pour soi, en fonction de son histoire. L'alcool vient dire chez chacun là où il en est dans sa souffrance. L'alcool, comme le suicide, est un fait social. Il concerne tout un chacun, de l'abstinent patenté à l'alcoolique notoire en passant par le buveur occasionnel. Nul n'y échappe. Chacun se positionne face à ce produit et trouve des réponses plus ou moins adaptées aux diverses sollicitations qui lui sont faites.

Les personnes en situation d'exclusion affichent là un certain cynisme salvateur. Elles nous convoquent à assister à notre débâcle. Elles nous montrent l'impuissance manifeste de nos stratégies sociales. Elles jouissent de nos incompétences à les empêcher de jouir, de notre propre renoncement pulsionnel. C'est pour cela que ces représentations vivantes du manque, nous sont généralement insupportables, et qu'il faut (par certains aspects) soit une bonne dose de masochisme soit un profond sadisme pour vouloir travailler avec ces personnes.

Entendons nous! Les professionnels ne sont pas des pervers diplômés. Lorsque je parle de masochisme, je prends cette notion dans ce qu'elle a de plus fondamentalement humain, qu'elle est au cœur de la constitution psychique et du symptôme, et qu'il n'est peut-être rien de plus symptomatique que le supposé choix d'un métier.

Pour les masochistes, ils y trouvent leur compte au quotidien. Eux aussi ont leurs rasades. Les exclus souffrent et font souffrir. Il n'est pas sans effet sur la psyché de travailler avec ces personnes. Ce texte même est à interroger dans ce sens. Mais pourtant, elles vivent, et c'est ce point-là qui peut nous faire revenir au travail chaque matin.

Il va s'en dire que les sadiques qui viennent là faire état de leur merveilleux altruisme, sont tout aussi malmenés. La victime est consentante... Pire, elle est déjà apprêtée, et soumise. Mais, heureusement, elle se dérobe, ne serait-ce pour montrer que toutes ces tentatives sont vaines et inefficaces.

Les personnes en situation d'exclusion présentent pour la plupart une forte propension au masochisme, dans le sens originel du terme. Là encore l'exclusion s'inscrit dans une histoire, et si les conditions sociétales contribuent largement aux processus de mise au ban, je considère qu'il ne faut en rien sous-estimer la part psychique qui s'y développe. Dans un précédent travail (Peraldi, 2006), j'ai pu souligner le fonctionnement masochiste qui sous-tend les processus psychiques liés à l'exclusion. Je ne voudrais pas trop reprendre ici ce que j'ai développé 
ailleurs mais, le masochisme, au cœur même du développement psychique de l'Homme, trouve son compte dans les phénomènes d'exclusion sociale, au point même que nul ne veut l'y voir. À l'instar de la pulsion de mort, il peut œuvrer en silence, entre le «pauvre enfant, qu'a-t-on fait de toi? ? de la compassion et le «ce n'est qu'un clochard» ou «un alcoolique » (selon le symptôme mis en avant) du volontarisme. Ce n'est bien sûr pas uniquement lié à la structure d'hébergement dans laquelle je travaille. Ce serait trop beau comme circonscription du problème, et trop effrayant pour travailler dans un tel lieu. Non, ces attitudes sont éminemment repérables dans toute structure où un individu est pris en charge, où il se donne à prendre en charge. Les fantasmes de fustigation sont très présents dans ce type de demandes. Rien que le nom de cette intervention pose des questions. On y sent tout le poids qui en résulte. C'est une charge à prendre à bras-le-corps, dans un corps à corps qui peut être parfois érotisé. Si je semble m'éloigner sensiblement de mon propos, c'est seulement un détour pour souligner la façon criante par laquelle ces personnes se délient du langage.

\section{IV}

Dubo, Dubon, Dubonnet...

Cette série phonétique qui m'a servi de point d'introduction est à lire dans ce hoquet, cette rupture dans le langage si présente chez les personnes en exclusion sociale et psychique. La langue boîte, elle se fait résistance au discours, elle décompose le lien social.

Précédemment, je parlais d'un état d'oubli du langage, ou du moins d'une réduction drastique du langage, comme pour ne plus pouvoir assumer le lien et la perte inhérents au langage. Il faut entendre ces hommes et ces femmes en état d'exclusion avant d'être en état d'ivresse. Au «qui suis-je?» qu'ils se posent et auquel nous devons parfois répondre, ils se demandent dans quels états ils errent. Jouons un peu sur les mots, surtout lorsque c'est le dernier «je» qu'il nous reste. Coluche, qui s'y connaissait en exclusion au point d'avoir créé les Restos du Cœur avec l'espoir fou que ce ne serait que provisoire - or, nous ne le savons que trop, le provisoire est fait pour durer - disait dans un de ses sketches : «Qui suis-je ? Où vais-je ? Dans quel état j'erre?» C'est indéniablement à ces trois questions que se confrontent les personnes en exclusion.

$\mathrm{Au}$ «qui suis-je ?», il est bien malaisé de répondre. Leur redonner un nom, c'est déjà les réinsérer dans le tissu social, mais aussi dans leur histoire: un patronyme est en même temps un toponyme qui ancre le sujet dans une généalogie mais aussi dans une situation géographique et historique. Loin d'être dans le déni, et sans pour autant que cela soit un refoulement, les personnes qui vivent à la rue, la bouteille à la main, ont coupé les amarres avec leur histoire. Elles se sont délestées de leur nom de famille souvent trop lourd à porter. Elles essayent au quotidien de sortir du symbolique pour s'ancrer jour après jour dans le réel. L'alcool les inscrit dans une certaine filiation. Il devient leur emblème, à tel point que l'on ne peut plus les écouter. Trop souvent j'ai entendu, à l'évocation d'un parcours d'errance, 
dire qu'untel racontait des histoires, voire qu'il était mythomane. L'alcool encadre la parole du buveur, dans sa forme comme dans son fond. Il la déforme pour qu'elle perdre son sens et son engagement. Cette question est, elle aussi, celle d'Hamlet. Dans le monologue de l'acte III, qu'il est inutile de présenter, le héros shakespearien tente de répondre à une insondable question existentielle :

«Être ou ne pas être. C'est la question.» (Shakespeare, 1957, 371)

La fin de cette tirade est moins connue. Néanmoins elle signe à merveille la position subjective d'Hamlet, bien sûr, mais aussi et surtout de la plupart des personnes en errance:

«Souviens-toi de tous mes péchés ».

Il faut prendre un certain temps pour bien mesurer tout le poids de cette demande. C'est éminemment là que vivent ces personnes à la rue, dans cet univers clos du péché. La morale et le volontarisme ne sont d'ailleurs pas en reste pour en rajouter. Les discours ambiants nous répètent, à tel point que cela en devient suspect, que les «exclus» et autres «SDF» sont soutenus par une logique sociale de l'assistanat, ou qu'ils n'ont pas de demande et que de ce fait rien ne peut être envisagé. C'est me semble-t-il une façon détournée de ne pas répondre, si ce n'est de maintenir l'écart creusé, au fil des jours et des bouteilles vidées, entre eux et nous. La demande si elle n'est pas, pour certains, explicite, n'en est pas moins criante: «Souviens-toi de tous mes péchés ».

«Où vais-je?» est une tout autre question. À partir du moment où ces personnes sont interpelées autrement que sur le mode de la rue et de la misère, l'errance, aussi pathologique soit-elle, est interrompue ne serait-ce que le temps de l'échange. Échanger, c'est donner un sens, à sa parole de prime abord, mais aussi à sa vie en général. Il y a un engagement par la parole, en cela que parler est toujours une prise de risque.

Le langage est une mise en relation, une véritable mise en mouvement, un jeu relationnel, où se déploie et se développe la perte et l'étranger en soi. N'y a-t-il pas, dans la clinique de la clochardisation, une nécessité à penser avec le « Je est un autre » de Rimbaud $(1972,250)$ qui permettrait de saisir l'autre qui parle en soi, notamment dans les phases d'alcoolisation. Cet aspect du «il», dont je parlais précédemment, qui vient enrayer tout discours possible sur l'alcool. Trop présent par son absence, ou trop absent dans sa présence, il n'est pas symbolisable de prime abord, mais conditionne toute relation aux autres tel le spectre du commandeur.

Le langage ordonne. Il classe et il décide. Mais il nous parle autant qu'il parle de nous. C'est à ce point d'orgue que je veux m'arrêter. En parlant avec, et j'insiste sur cette préposition, nous donnons une direction au langage, nous l'ordonnons vers un autre à soi, un autre en soi. Dans ce cas, si ma supposition n'est pas erronée, l'échange ouvert par la parole crée de nouvelles perspectives. À ce « où vais-je?» répond un «où la parole vous mènera», «où nos paroles nous mèneront ». Ceci n'est pas sans faire écho à la citation de Freud qui, pour décrire l'inconscient, reprenait à son compte le principe d'Itzig, le cavalier du dimanche: 
«Où vas-tu donc, Itzig?», «Moi, je n'en sais rien. Interroge mon cheval!» (Freud, 1898, 229).

La psychanalyse, cette écoute de l'inconscient,

«intervient là pour briser ce cercle de la parole de la misère à la misère de la parole» (Assoun, 1999, 22).

La parole de la misère, c'est celle du préjudice, de la responsabilité imputée à l'autre. Si elle indique où en est un sujet dans son rapport au monde, elle étouffe pour autant ce qu'il en est de lui; elle signifie quelque chose mais pas forcément quelqu'un. La misère de la parole, c'est celle de l'injustice, d'un «je» qui essaye de se définir dans son histoire et à partir de son histoire, d'une vérité qui cherche à se dire. Or, la vérité ne s'oppose pas au faux mais à l'oubli. C'est de cet oubli de soi, de cette parole qui déserte la subjectivité dont il est éminemment question avec ces personnes à la rue. Parler avec elles, c'est lutter contre l'oubli.

Dans ma pratique, un des points d'achoppement est bel et bien de retracer le cours des événements, non pour y trouver une illusoire causalité mais pour mieux cerner les impasses subjectives. Pour beaucoup de personnes en exclusion, leur histoire commence au moment de la rupture, quelle soit sociale, familiale, sentimentale ou locative. Quid de ce qui se passait avant? Le point d'entrée dans le discours est la rue. Le problème est qu'il s'agit aussi du point final. Plus d'avant, et pas d'après. Le temps se conjugue au présent. "Godot arrive demain». C'est une des difficultés majeures de toute prise en charge possible de la souffrance d'une personne. Il nous faut travailler au présent, au jour le jour, pour qu'à un moment quelque chose du passé revienne dans le discours, sans être traumatique. C'est un véritable jeu dans les temps, une mise en mouvement des temporalités qui se fait et se défait tant que la relation n'est pas suffisamment contenante pour s'engager dans un travail d'élaboration.

Ceci n'est pas sans évoquer le laborieux tissage de Pénélope. Rappelons-nous : alors qu'elle attendait son époux, l'ingénieux Ulysse, Pénélope était avidement sollicitée par de nombreux prétendants. Pour se protéger et en même temps répondre aux efforts de ces illustres personnages, elle avait promis qu'elle en épouserait un lorsqu'elle aurait fini de tisser le linceul de son père. Elle commença ainsi son ouvrage, espérant que son cher et tendre rentrerait avant l'heure dite. Or, les jours passaient. Ulysse étant toujours aux abonnés absents, Pénélope s'évertua donc à tisser à lumière du jour, pour mieux le soir tombé défaire ce qu'elle avait créé. Cela lui permis ainsi de retarder un mariage malheureux.

Faire référence à l'Odyssée homérique, c'est une façon imagée de tisser, si je puis dire, ce qu'il en est du rapport au temps dans lequel vivent ces personnes en errance, et par conséquent du travail possible à faire avec elles. D'un côté, elles ne s'inscrivent pas dans un présent, mais bien dans un passé, un temps figé, mort. De l'autre, elles font et défont au quotidien ce qui fait leur histoire et, bien sûr, ce qui 
est fait pour rompre les chaînes de l'exclusion. Si elles retardent ce qui paraît inéluctable, c'est toujours d'un linceul dont il est question. La pulsion de mort, cette notion si chère à Freud et si problématique pour ses disciples à tel point que certains préférèrent qu'elle n'existe pas, règne quasiment sans partage. Elle est au cœur de la psyché, se développe insidieusement dans les institutions et se distille dans les faits (l'effet) de société.

Ce qui est important à prendre en compte, comme un repérage clinique, c'est que ces personnes (se) vivent dans un profond oubli. Cet oubli, c'est avant tout celui recherché dans l'alcool. On l'entend souvent, elles boivent pour oublier. Mais force est de constater qu'alcoolisées, elles n'oublient rien, elles se rappellent encore mieux. Les souvenirs viennent comme une lame de fond terrasser les résidus du Moi. Comme cela ne fonctionne donc pas, elles vont chercher l'oubli au plus profond d'elles-mêmes, au tréfonds, là où elles se perdent souvent sans espoir de retour. Cela se manifeste dans l'oubli du corps. Les personnes à la rue se vivent dans une profonde analgésie.

«Tu es ceci» écrivait très justement Lacan parlant du réel du corps qui se fait maladie. La «chair en tant qu'elle est souffrante, qu'elle est informe, que sa forme par soi-même est quelque chose qui provoque l'angoisse.» (Lacan, 1954-1955, 186)

Elles ne ressentent plus ce corps qui se délite progressivement, qui s'éparpille sur la chaussée, qui se vide jusqu'à ne plus jamais pouvoir se remplir. C'est un panier percé. Cette expression, normalement, est appliquée aux personnes qui dépensent leur argent sans faire attention, sans réfléchir dit-on. Les personnes en exclusion aussi dépensent sans compter, ni ce que cela coûte ni le temps que cela prend. L'équivalence entre l'argent et les excréments n'est plus à faire. Après le porte-monnaie, c'est le corps qui se vide, qui se laisse aller, jusqu'à renier, dans un ultime effort, le rythme biologique. La pulsion de mort est encore en œuvre...

C'est dans cet état d'oubli de soi, ou pour faire un néologisme, d'oubliance, comme un processus jamais achevé, qu'elles errent. Quels hères! Nous sommes les jouets participants de leurs errances, du bal des mis au ban transbahutés par les bus là où le regard ne porte pas. Cette oubliance c'est une sortie du symbolique, c'est un passage à l'acte au ralenti qui prend forme jour après jour. Le langage se replie sur lui-même. L'on repère un processus de désexualisation du lien de la parole. Pas de «je», pas de «tu» et presque plus de «il » ${ }^{10}$. Reste un «on» qui vient (indé) finir la relation à l'Autre. Cette jouissance, parce que c'en est une, marque dans son lien étroit à l'alcool la prévalence d'une fixation orale qui prête à la représentation imaginaire d'un flot, d'un flux liquide et non-verbal, hors discontinuité, hors limites. La perte du langage joue de ce retour à l'infans et au besoin. Dans les alcoolisations massives des personnes en exclusion, le besoin vient noyer le désir. Ces personnes ont physiquement besoin de boire, à heure répétée. Le rythme des bouteilles succède à celui des tétées. L'alcoolique sur son banc ne parle pas, il éructe. En se désinscrivant de la langue, la personne en exclusion (a) boit. Le (a) est à entendre là de différentes façons. Ce (a) c'est celui du verbe avoir qui se conjugue au devoir. «Parce que c'est ce qu'on attend de moi» répondait Dylan Thomas 
lorsqu' on lui demandait pourquoi il buvait. Une justification peut-être, un processus d'identification certes, mais aussi un jeu où réalité psychique et réalité extérieure, pour reprendre ces catégories freudiennes, se rencontrent et se confrontent. Mais c'est bien évidemment aussi l'objet a, mis en exergue par Lacan. Il est privatif parce qu'on ne le retrouve jamais, bien que l'on passe sa vie à le chercher. Il n'est pas dans la première gorgée, et encore moins dans la dernière, lorsqu'on voit «le cul» de la bouteille (tout le poids du sexuel dans le rapport à l'alcool se développe dans cette expression). Il est privatif parce qu'il plie le langage à cette bouteille explosée sur un mur, à cette canette broyée sur la chaussée. Si l'Homme est un être dénaturé par le langage, l'exclusion et l'alcoolisation, qui vont de pair, animalisent le «sujet». C'est là tout un enjeu, dans la clinique de la désocialisation. La passion qui anime l'alcoolique pour son objet, tout comme celle qui le met au ban, exclut la référence à un tiers. Inviter ces personnes à parler participe d'une position clinique que Lacan soutenait être une clinique du sujet.

«À quoi ça sert le langage? » demandait-il, «il fait le sujet» (Lacan, 1967).

Le travail possible est de tenter de retrouver ce noyau de vérité historique et de donner au sujet les chances d'une symbolisation. Les amener à accepter qu'il y ait du vide, un manque structurel qui ne puisse pas être comblé. Combler le manque, c'est bien ce qu'ils payent.

Spécifier un lieu pour accueillir ces sujets errants, réticents aux normes sociales, c'est se donner les moyens de regarder enfin ailleurs; ailleurs et autrement, même si nos stratégies viennent se heurter à leurs jouissances qui s'inscrivent aisément dans le réel, surtout lorsque la réalité le leur permet, les y autorise, si ce n'est les y encourage. «Wo Es war soll Ich werden» (Freud, 1984, 110) «Là où C'était, Je dois advenir», pourrait-on traduire. On passe de l'indéfini, du pulsionnel à la subjectivité, au sujet. C'est le travail civilisateur. C'est redonner une dimension, une dignité à ces personnes, les inclure dans le lien social en tant qu'il passe par le langage, le leur et le nôtre. C'est faire le pari qu'il y a une autre inscription possible pour ces personnes, un espace intermédiaire entre la jouissance de la rue et les exigences de la société, la possibilité d'une jouissance qui soit tenable.

\section{V}

C'est à ce point, me semble-t-il, que s'articule ce que j'appellerais aujourd'hui ces lois du réel. C'est bien d'abord de la loi de l'inconscient, qui se noue en fonction des trois registres évoqués précédemment. Puis, celle du corps qui vient saisir un sujet dans sa propre histoire, lui signifier comment sa jouissance s'organise et se joue du travail civilisateur. Et puis la dernière, mais non la moindre, celle de l'économie de marché. Elle n'est pas indépendante des sujets qui s'y confrontent. Elle se développe, mute si je puis dire, en fonction d'une société constituée d'une pluralité de personnes à un moment donné de son histoire. Cette économie sociétale est à prendre en ce qu'elle renvoie de plein fouet à celle du psychisme. Les constructions psychiques sont dépendantes des contextes historiques, des événements traversés, des idéologies, de diverses influences. Les 
pathologies ne cessent d'évoluer. On sait depuis Freud que l'inconscient ignore le temps, mais il n'est pas dit que le temps ignore l'inconscient (Dufour, 2003, 41).

Le langage est le signe manifeste d'une perte inaugurale. Il nous met en lien parce que nous sommes désespérément à la recherche d'un objet, perdu et jamais totalement retrouvé. Lorsque la société vient boucher tout manque à être, cela ne peut être sans effet sur la subjectivité des sujets qui la composent. C'est ainsi que je comprends la clinique de la clochardisation, ou de l'exclusion, comme une véritable clinique du réel. C'est à cela que le praticien avec un patient, un résidant, ou un usager, selon le terme choisi, a à faire. Il ne peut pas en faire l'impasse.

Pour contrer le réel, pour donner du sens à ces fractures du quotidien, le praticien, et ce, quel que soit son champ d'interaction, doit user de tout le symbolique possible, donc tout le langage possible. C'est là une nécessité pour travailler avec ces personnes. Trouver un au-delà qui ne soit plus une ivresse pathologique, un reniement du corps ou une soumission quasi totale à la loi de la consommation. Pouvons-nous trouver d'autres assentiments? Refaire le monde, c'est bien ce que tente de faire celui qui boit. C'est sur cet écart, entre soi et la perception de soi, qu'ouvre l'ivresse, que nous pouvons travailler.

Écouter les mots de la rue, c'est contrecarrer le silence assourdissant de l'exclusion. C'est encore une position médiane, si ce n'est tierce, entre l'abandon et le symptôme. C'est être cet autre qui fait signe. La psychanalyse a là une place importante à jouer pour contrecarrer certaines positions dogmatiques liées au discours idéologique du moment. La psychanalyse, comme lecture du sujet en devenir, ne peut pas être sans voix quant au lien social et au vivre ensemble. Ne peut-on pas, avec Lacan, la considérer comme une boussole efficace dans le champ de la direction de l'éthique? (Lacan, 1959-1960) Elle mesure les effets de la société et de ses mutations, ne serait-ce lorsque le réel social surgit souvent avec force. Le sujet se construit en fonction des objets auxquels il se confronte. L'alcoolisme d'un père ou d'une mère ne conditionne pas nécessairement celui d'une fille ou d'un fils. Il en est une répétition, la trace. Trace subjective bien évidemment, mais aussi trace d'une réalité qui est venue obstruer toute autre potentialité. Nous devons prendre en compte le parallèle important qui existe entre la communauté humaine et le fonctionnement psychique individuel. C'est s'inscrire dans la continuité du travail de civilisation tel qu'il a été pensé par Freud (Freud, 1929). Je considère qu'il est nécessaire, du moins avec cette frange de la population qui vit à la rue, stigmatisée par l'alcoolisme, de les entendre dans leur «corps social» pour, à l'image du sculpteur de marbre, les aider conjointement à se (re) façonner un «corps individuel». C'est dans cette dynamique qui se tient mon propos, dans ce lien toujours malmené, tant d'un côté que de l'autre, entre réalité psychique et réalité extérieure.

nicolas peraldi

8 , rue de belzunce

75010 paris

nicolas.peraldi2@free.fr 


\section{Notes}

1. Comment comprendre le paradoxe français? Des études faites sur un échantillon de la population de Bordeaux et de Manchester ont prouvé que boire du vin était meilleur pour la santé que la bière et qu'il y a avait moins d'accident cardio-vasculaire dans le Sud de la France que dans le Nord de l'Angleterre. Quid du fait que la culture de la vigne est profondément ancrée dans l'histoire de la Gironde, et que la bière fait partie des aliments de base des pub anglais. Quid du fait que Bordeaux est une ville riche alors que Manchester souffre depuis longtemps d'un haut taux de chômage. Quid du fait que Bordeaux se situe dans une région ensoleillée, agréable à vivre, alors qu'à Manchester, s'il fait beau, c'est plusieurs fois par jour...

Comment entendre aussi ce candidat UMP à la présidentielle 2007, connu pour être abstinent, qui déclare un verre de Sancerre à la main qu'il faudrait modifier la loi Evin parce que le vin n'est pas une drogue ? Ou cet autre candidat à la présidentielle, soutenu par le Parti des Travailleurs, qui dans une émission politique matinale déclare sans ambages, Pasteur comme référence, que le vin ce n'est pas de l'alcool...

Et cette publicité des «6 organisations professionnelles les plus représentatives de la filière des boissons alcoolisées » (www.2340.fr) promouvant dans les grands quotidiens français le nombre possible de verres à boire par jour lorsque l'on est une femme, un homme,... Fait intéressant à noter, en deçà des singularités physiologiques autres que la différence des sexes, la quotidienneté à été supprimée des facteurs à risques.

2. Je développerai tout au long de cette élaboration différents aspects de cette notion qu'ils soient psychanalytiques, anthropologiques et sociologiques.

3. Centre d'Hébergement et de Réinsertion Sociale. Le terme de réinsertion est bien évidemment plus à entendre comme un idéal administratif que comme une réalité. Comment, et pourquoi d'ailleurs, réinsérer des personnes qui pour la plupart n'ont pas été insérées?

4. Cette expression est assez significative de la façon dont sont perçues les personnes en exclusion puisque dans cette tautologie l'adjectif redouble le nom.

5. Expression métaphorique populaire. Le ballon, c'est l'éthylotest («souffler dans le ballon»), c'est le car de police (ou «panier à salade»), et c'est enfin la prison (où la seule activité proposée était de jouer au football).

6. J'utilise le terme de résidant et de référant sous la forme d'un participe présent pour signifier la part active qui se joue dans cette relation.

7. Je pense notamment à ce résidant hospitalisé pour une maladie infectieuse qui se retrouva dès lors sevré d'alcool. Pendant plusieurs semaines, il resta alité, surpris lui-même de ne pas sortir boire un verre. La problématique de l'arrêt de l'alcool était bien évidemment au cœur même de ses questionnements et ce avant qu'il ne soit hospitalisé. Mais du jour au lendemain, il n'y aurait jamais pensé. Quoi qu'il en soit, au fil de nos entretiens, l'alcool était toujours aussi présent dans son discours. L'absence se faisait amèrement ressentir. Tous les jours, «trois fois par jours », il pensait à l'alcool : à chaque fois qu'il finissait un des trois quotidiens gratuits que l'infirmière lui amenait chaque matin. En quatrième de couverture, pour tous les périodiques, une marque de bière faisait l'éloge de son système particulier de pression à installer chez soi...

8. Ce texte fut écrit quelques mois avant l'apparition des Enfants de Don Quichotte et du campement du Canal Saint Martin. Je me suis expliqué sur leur action en faveur des «SDF» (Libération, Rebonds, 23.01.07). Si nos références littéraires sont les mêmes, bien que j'opterais plus pour le rôle de Sancho Pansa — analyste avant l'heure —, je maintiens encore aujourd'hui que de vouloir travailler avec ces personnes en exclusion n'est pas sans effets subjectifs, et qu'il faut se méfier des bonnes intentions. Les difficultés auxquelles les militants du Canal sont confrontés à l'heure où je relis ces lignes ne font que confirmer mon assertion.

9. Je fais ici référence aux différents travaux de D.R. Dufour. Loin de tout moralisme, en philosophe, il pose un regard critique sur les dérives de notre société néo-libérale, et ses multiples effets sur le processus de subjectivation.

10. Je renvoi là encore aux travaux de D.R. Dufour avec le Mystère de la trinité.

\section{Bibliographie}

Assoun, P.L., 1999, Le sujet du préjudice: l'«exclusion» à l'épreuve de la psychanalyse in Dire l'exclusion, F. De Rivoyre (sous la direction de), Ramonville, Ed. Eres, 15-29. 
Dubo... Dubon... Dubonnet. Lecture des problématiques alcooliques aux regards des faits de société

Dufour, D.R., 2003, L'art de réduire les têtes, sur la nouvelle servitude de l'homme libéré à l'ère du capitalisme total, Paris, Ed. Denoël.

Duras, M., 1985, Extrait d'une interview à France Culture, Alcoolisme: approche psychanalytique, Bulletin de l'Association freudienne en Belgique, $\mathrm{n}^{\circ} 4$.

Freud, S., 1898, Lettre $\mathrm{n}^{\circ} 92$ in Naissance de la psychanalyse, Paris, Presse universitaire de France, 1986.

Freud, S., 1929, Malaise dans la culture, Paris, Presse Universitaire de France, Col. Quadrige, 2005.

Freud, S., 1933, «La décomposition de la personnalité psychique», XXXI conférences in Nouvelles conférences d'introduction à la psychanalyse, Paris, Gallimard, 1984.

Gentis, R., 1971, Guérir la vie, Col. Textes à l'appui, François Maspero, Paris.

Lacan, J., 1953, «Le symbolique, l'imaginaire et le réel», in Des Noms-du-père, Col. Comment faire pour enseigner la psychanalyse, Éd. du Seuil, Paris, 2005.

Lacan, J., 1954-1955, Le Moi dans la théorie de Freud et dans la technique de la psychanalyse, Séminaire II, Paris, Ed. du Seuil, 1978.

Lacan, J., 1959-1960, Ethique de la psychanalyse, Séminaire VII, Paris, Éd. du Seuil, 1986.

Lacan, J., 1967, Petit discours aux psychiatres, inédit.

Peraldi, N., 2006, Boiter n'est pas un péché. L'exclusion comme enfermement, Esprit, nº 12, 22-44

Perrier, F., 1982, L'alcool au singulier, l'eau de feu et la libido, Paris, Inter Éditions.

Rimbaud, A., 1972, Lettre à Paul Demeny du 15.05.1871, Euvres complètes, Bibliothèque de la Pléaïde, Paris.

Shakespeare, 1957, Hamlet, Acte 3, Scène 1, vers 56-90, Paris, Euvres complètes, Éd. Bilingue Le Club Français du Livre.

Winnicott, D.W., 2000, «La crainte de l'effondrement», La crainte de l'effondrement et autres situations cliniques, Paris, Gallimard, Col. Connaissance de l'inconscient. 